\title{
Chlorosulfuric acid-assisted production of functional 2D
} materials

\author{
Mohsen Moazzami Gudarzi (D) $^{1,2}$, Maryana Asaad ${ }^{1,2}$, Boyang Mao ${ }^{2,3}$, Gergo Pinter iD $^{1,2}$, Jianqiang Guo ${ }^{4,5}$, Matthew Smith ${ }^{1}$, \\ Xiangli Zhong (iD), Thanasis Georgiou ${ }^{6}$, Roman Gorbachev $\mathbb{D}^{2,3}$, Sarah J. Haigh $\mathbb{D}^{1,2}$, Kostya S. Novoselov ${ }^{3,7}$ and Andrey V. Kretinin (D) $^{1,2 凶}$
}

The use of two-dimensional materials in bulk functional applications requires the ability to fabricate defect-free 2D sheets with large aspect ratios. Despite huge research efforts, current bulk exfoliation methods require a compromise between the quality of the final flakes and their lateral size, restricting the effectiveness of the product. In this work, we describe an intercalation-assisted exfoliation route, which allows the production of high-quality graphene, hexagonal boron nitride, and molybdenum disulfide 2D sheets with average aspect ratios 30 times larger than that obtained via conventional liquid-phase exfoliation. The combination of chlorosulfuric acid intercalation with in situ pyrene sulfonate functionalisation produces a suspension of thin large-area flakes, which are stable in various polar solvents. The described method is simple and requires no special laboratory conditions. We demonstrate that these suspensions can be used for fabrication of laminates and coatings with electrical properties suitable for a number of real-life applications.

npj 2D Materials and Applications (2021)5:35; https://doi.org/10.1038/s41699-021-00215-2

\section{INTRODUCTION}

Graphene and other two-dimensional (2D) materials are gradually finding their way into real-life applications, including nanocomposites and biomaterials, energy and microelectronics ${ }^{1-4}$. Some of the most promising application areas use 2D materials to produce membranes for water desalination ${ }^{2,3}$, or laminates for thermal management and microwave shielding ${ }^{4}$ and conformal coatings for wearable electronics ${ }^{5}$. All of these applications rely on the bulk synthesis of large-area high-quality flakes of 2D material, most commonly graphene, hexagonal boron nitride $(\mathrm{hBN})$, or transitional metal dichalcogenides (TMDs).

The classical top-down approach of micromechanical cleaving is incompatible with large-scale production, and bulk synthesis of 2D materials is dominated by alternative exfoliation methods such as chemical $^{6,7}$, liquid-phase (LPE) ${ }^{8,9}$, intercalation-assisted ${ }^{10-15}$, and electrochemical $^{16,17}$. Unfortunately, each of these alternatives requires a compromise between the quality of the final flakes and their lateral size ${ }^{1,18}$.

Recently, a method previously used for dispersion of carbon nanotubes ${ }^{19}$ in superacids such as chlorosulfuric acid $(\mathrm{CSA})^{20}$ was utilised for the production of $2 \mathrm{D}$ materials. It was suggested ${ }^{21}$ that layered materials undergo intercalation and subsequent protonation in CSA. As a result of protonation, a strong interlayer electrostatic repulsion is developed, which triggers spontaneous dissolution (exfoliation). This approach has been successfully applied for exfoliation of graphene ${ }^{21}$, hexagonal boron nitride $(\mathrm{hBN})^{22}$, and various transitional metal dichalcogenides ${ }^{23,24}$.

Despite the promising combination of the quality and large size of the flakes obtained through this method, it has a significant drawback; the stability of the CSA dispersions depends strongly on the acid strength. Trace amounts of moisture are sufficient to deprotonate the 2D material surface, causing reaggregation and restacking ${ }^{21}$. Therefore, further processing to specific applications is limited by the need to handle the acid dispersions in a tightly controlled environment ${ }^{19}$.

In this work, we modified the superacid intercalation method ${ }^{21-24}$ to make the resultant dispersions compatible with different organic solvents and stable in ambient environmental conditions. The idea of the suggested technique is to take advantage of the CSA's excellent ability to intercalate, sacrificing the possibility of spontaneous exfoliation for processing flexibility. Instead, we in-situ functionalised the 2D material dispersed in CSA with the derivatives of pyrene sulfonic acid so that the dispersion retains its colloidal stability in a more suitable solvent. The pre-intercalated and functionalised bulk crystal is subsequently mechanically exfoliated in a brief burst of ultrasound and homogenised by shear force mixing. The limited duration of the mechanical impact ensures a low level of structural damage and produces thin sheets with a large aspect ratio. To assess the properties of the resulting materials, we fabricated large-area high-quality flexible laminates of graphene and hexagonal boron nitride and characterised their electrical properties.

\section{RESULTS AND DISCUSSION} Exfoliation procedure

To tackle the poor processability of $2 \mathrm{D}$ materials produced by exfoliation in CSA, we simultaneously exfoliate and non-covalently functionalise the layered material. Chlorosulfuric acid is a renowned sulfonating reagent for pyrene ${ }^{20}$, so by adding a small amount of pyrene to CSA, we produce a self-contained mixture of the intercalant and pyrene tetra sulfonic acid, which acts as the stabilising agent ${ }^{25}$ and is the product of the sulfonation reaction in excess CSA ${ }^{20}$

In our method, the bulk crystal (graphite, hBN, or molybdenite) was stirred with the CSA/pyrene mixture for $48 \mathrm{~h}$ at room temperature under ambient conditions. This stirring facilitated

\footnotetext{
${ }^{1}$ Department of Materials, School of Natural Sciences, The University of Manchester, Manchester, UK. ${ }^{2}$ National Graphene Institute, The University of Manchester, Manchester, UK. ${ }^{3}$ Department of Physics and Astronomy, School of Natural Sciences, The University of Manchester, Manchester, UK. ${ }^{4}$ AECC Beijing Institute of Aeronautical Materials, Beijing, China. ${ }^{5}$ Beijing Institute of Graphene Technology, Beijing, China. ${ }^{6}$ The Henry Royce Institute, The University of Manchester, Manchester, UK. ${ }^{7}$ Department of Materials Science and Engineering, National University of Singapore, Singapore, Singapore. ${ }^{凶}$ email: andrey.kretinin@manchester.ac.uk
} 
a

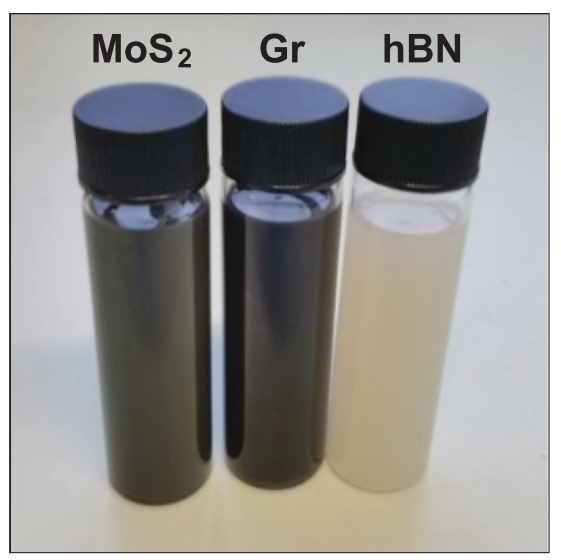

\section{b Energy (eV)}

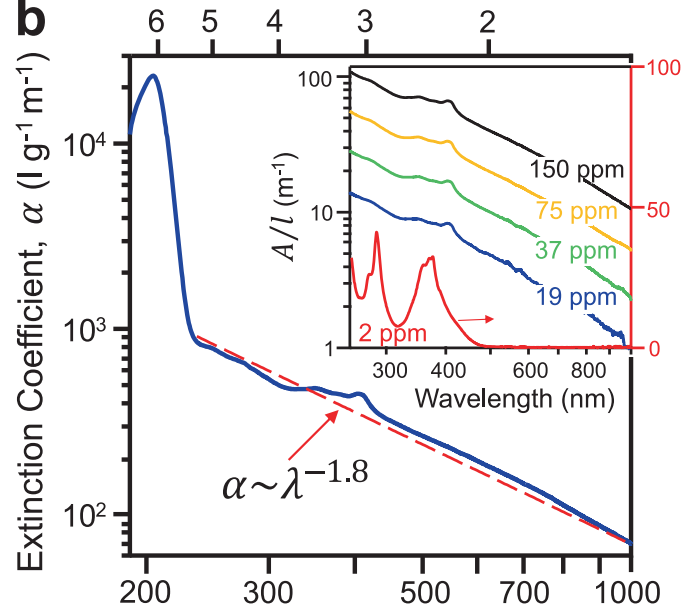

Wavelength $(\mathrm{nm})$
C

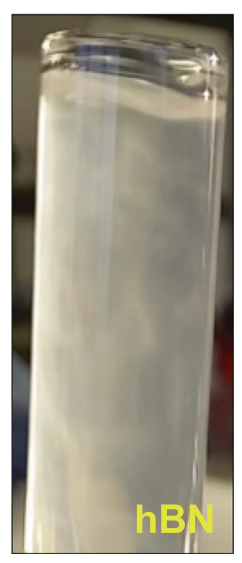

Fig. 1 Superacid assisted exfoliation of the large-area solution-processable 2D materials. a Photograph of exfoliated of MoS ${ }_{2}$, graphene, and hBN nanosheets dispersed in N-methyl-2-pyrrolidone (NMP) at a concentration of around $0.5 \mathrm{~g} \mathrm{I}^{-1}$. $\mathbf{b}^{\text {The Ultraviolet-Visible (UV-Vis) }}$ absorption spectra of the aqueous dispersions of exfoliated hBN 2D sheets represented as the extinction coefficient $a$ versus the wavelength $\lambda$ (blue curve). The spectrum demonstrates the absorption onset at around $6 \mathrm{eV}$, corresponding to the hBN bandgap. The red dashed line is the power-law approximation of the extinction coefficient. The feature in the spectrum between 300 and $500 \mathrm{~nm}$ is associated with absorption by the pyrene sulfonate functionalisation molecules. Inset: The spectra of the absorption coefficient $A$ per length $I$, of the same hBN dispersion taken at different nanosheet concentrations (blue, green, orange and black curves). The red curve is the reference absorption spectrum of the pyrene sulfonate dispersed in the water showing typical absorbance in the UV region. c Photograph of hBN nanosheet dispersion in NMP $\left(0.1 \mathrm{~g} \mathrm{I}^{-1}\right)$ illustrating the shear-induced birefringence visible as a colour hue.

intercalation of the layered crystal in the presence of the functionalising molecules. Contrary to previous reports with only $\mathrm{CSA}^{21-24}$, we did not observe spontaneous exfoliation of the bulk material, most likely due to the protonation being suppressed by the insufficient strength of CSA. To proceed with exfoliation, the acid-treated material was thoroughly washed in deionised water and transferred to N-methyl-2-pyrrolidone (NMP) or dimethyl sulfoxide (DMSO) at a concentration of $5 \mathrm{gl}^{-1}$. The actual exfoliation was triggered by ultrasound energy delivered in an ultrasonic bath followed by high shear mixing. For each step, the exposure to mechanical impact never exceeded 10-15 min and produced NMP or DMSO dispersions with excellent colloidal stability (Fig. 1a).

Despite the short mechanical exfoliation step, the yield of exfoliated material was noticeably higher than the value reported for the conventional LPE method under similar processing conditions (Supplementary Figs $1-3)^{8}$. The highest value was obtained for $\mathrm{MoS}_{2}(\approx 8 \mathrm{wt} \%)$, followed by graphene $(\approx 5 \mathrm{wt} \%)$ and hBN $(\approx 1$ wt $\%)$. Here, the yield is determined as the amount of solid material remaining after final centrifugation (see "Methods" section). The cumulative effect of the CSA and sulfonated pyrene stabiliser on the exfoliation rate is particularly crucial for this method. Excluding pyrene from the CSA intercalation or application of the conventional LPE method in the presence of the sulfonated pyrene resulted in a dramatic decrease of the yield. By using finer powders of bulk graphite $(<20 \mu \mathrm{m})$, it was possible to increase the graphene yield up to $18 \mathrm{wt} \%$, at the expense of the final flake size (Supplementary Fig. 5). However, regardless of the size of the initial layered crystals, the omission of CSA intercalation failed to produce exfoliated 2D sheets larger than $1 \mu \mathrm{m}$.

\section{Physical and chemical characterization of the exfoliated material}

To characterise the resulting flakes, we first assessed the absorbance spectrum of the aqueous dispersion of hBN (Fig. 1b). The measured extinction coefficient, $a$, demonstrated the power-law dependence on the photon wavelength $\lambda\left(a \propto \lambda^{-1.8}\right)$, which is consistent with non-resonating light scattering ${ }^{26}$. The observed value of $a \approx 533$ I $\mathrm{g}^{-1} \mathrm{~m}^{-1}$ at $\lambda \approx 300 \mathrm{~nm}$ is significantly lower than that reported for hBN dispersions obtained via a conventional LPE method ${ }^{8}$. This result, as well as the value of the scattering exponent of around 1.8, which is indicative of the van de Hulst regime ${ }^{26}$, confirms the dominance of large yet thin $2 \mathrm{D}$ sheets. The apparent large lateral size of the $2 \mathrm{D}$ sheets made it possible to visualise them using optical microscopy (Fig. 2a-c). We performed a statistical analysis of the lateral size and thickness measured by atomic force microscope (AFM) (Fig. 2d-f). The log-normal distribution gives a good approximation for both the lateral size and the number of layers, which is a sign of random fragmentation of a larger bulk crystal ${ }^{27}$. For graphene and hBN, the average thickness was about 6-7 layers with a mean lateral size of $6 \mu \mathrm{m}$ and $4 \mu \mathrm{m}$, respectively. The average number of layers for $\mathrm{MoS}_{2}$ nanosheets was $\sim 13$, with a mean lateral size of $5 \mu \mathrm{m}$. Figure $2 \mathrm{~g}-\mathrm{i}$ compares our data with the previously published work. It is evident that for all three 2D materials, our exfoliation method provides previously inaccessible range of sizethickness values, namely few-layer flakes with the lateral dimension between 1 and $10 \mu \mathrm{m}$. To complement the size distribution data Fig. $2 \mathrm{j}-\mathrm{I}$ shows low-magnification transmission electron microscopy (TEM) images of the flakes together with the corresponding electron diffraction patterns indicating high crystallinity, with no substantial damage introduced by the acid pre-treatment (Supplementary Figs 21-23).

The produced 2D sheets are functionalised with pyrene molecules. For the aqueous dispersion of hBN, this is demonstrated by the spectral features at around $300-400 \mathrm{~nm}$ in the absorption spectrum (Fig. 1b), which are typical for sulfonated pyrene $^{28}$. It is worth-noting that these are not attributable to the solvent as this was pure deionised water. For graphene, the pyrene functionalisation is visible in the Raman spectrum (grey trace in Fig. 3a), which has a series of additional pyrene-attributed peaks between 1100 and $1450 \mathrm{~cm}^{-1}$ that interfered with the Dpeak of graphene (Supplementary Fig. 24). In order to eliminate these and measure the quality of the material, the pyrene functionalisation was decomposed by annealing at $1100^{\circ} \mathrm{C}$. After 

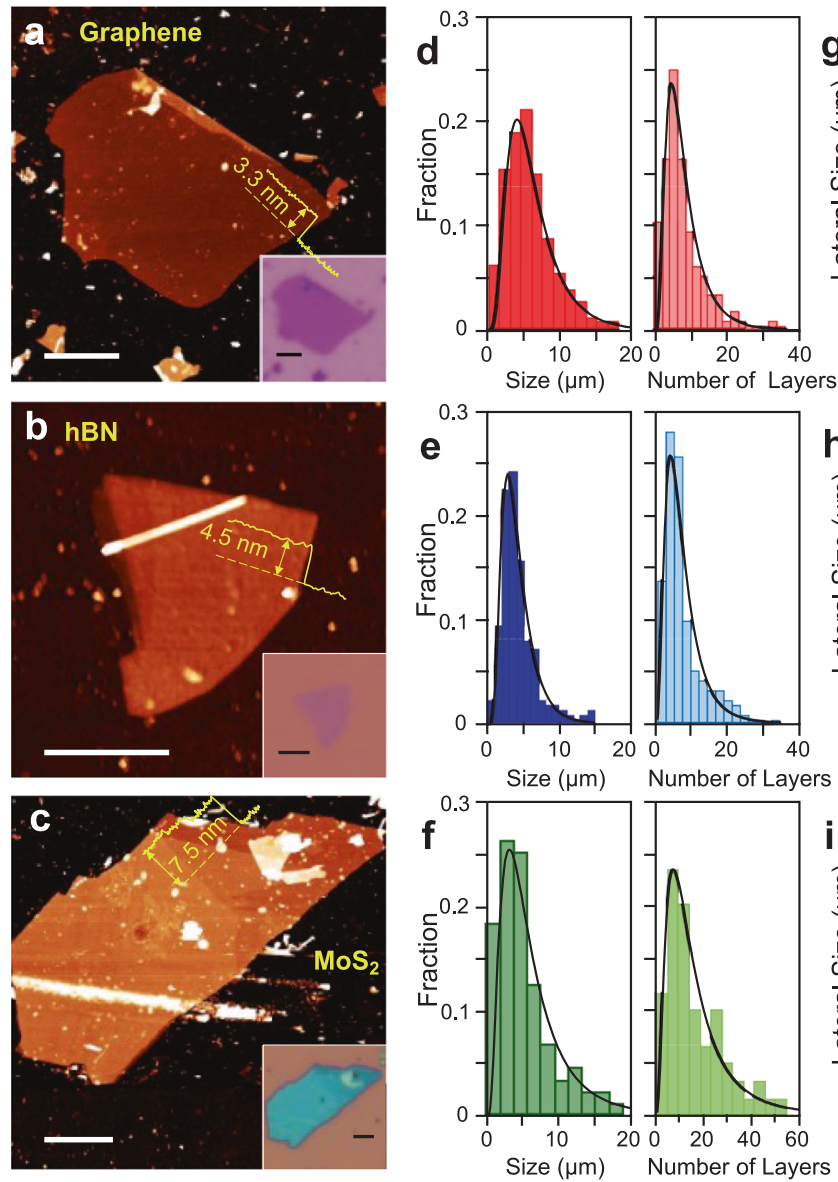
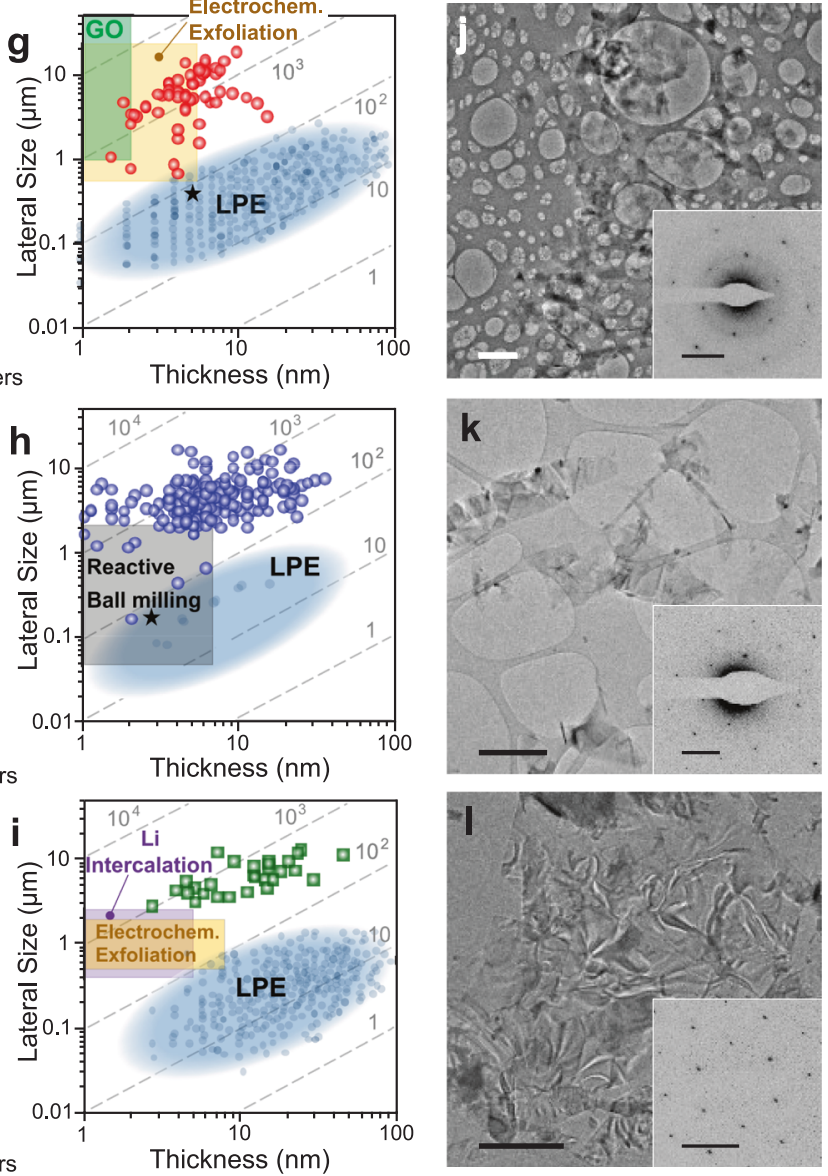

Fig. 2 The size distribution of the exfoliated 2D sheets. a-c AFM images of graphene (a), hBN (b), and MoS 2 (c) 2D sheets with the corresponding height profiles (yellow traces). Inset to panels a-c: Optical micrographs of the same 2D sheets under white light atop of a Si/ $\mathrm{SiO}_{2}$ substrate with a $290 \mathrm{~nm}$-thick thermal oxide. Scale bars: $2 \mu \mathrm{m}$. d-f Histograms demonstrating the distribution of the lateral size (defined as the square root of the nanosheet area) and the number of layers for graphene (d), hBN (e), and MoS $_{2}$ (f) $2 \mathrm{D}$ sheets. Black solid curves in $\mathbf{d}-\mathbf{f}$ represent the approximation of the experimental data with the log-normal distribution function ${ }^{27}$. $\mathbf{g}$-i Comparison of the nanosheet lateral size versus thickness with the values reported for other exfoliation methods. The borders of the rectangles/ellipses are the reported upper and lower limits. The dashed grey lines mark the corresponding aspect ratio. g Exfoliated graphene: red circles-present work, orange rectangleelectrochemical exfoliation ${ }^{16,17}$, green rectangle-chemical exfoliation including $\mathrm{GO}^{6,7}$ and chemical intercalation ${ }^{11}$, black star-LPE in the presence of aromatic agents ${ }^{46}$, dark blue ellipse/circles-conventional LPE ${ }^{9,18} \cdot \mathbf{h}$ Exfoliated hBN: blue circles-present work, grey rectanglereactive ball milling ${ }^{47,48}$, black star-LPE in the presence of aromatic agents ${ }^{49}$, dark blue ellipse/circles-conventional LPE ${ }^{8,18}$. i Exfoliated $\mathrm{MoS}_{2}$ : green squares-present work, orange rectangle-electrochemical exfoliation ${ }^{13}$, purple rectangle-lithium intercalation ${ }^{12}$, dark blue ellipse/circles-conventional LPE ${ }^{18,50}$ (See Supplementary Table 1). j-I Low-magnification transmission electron micrographs of large graphene (g), hBN (h), and $\mathrm{MoS}_{2}$ (i) 2D sheets. Scale bars: $2 \mu \mathrm{m}$. Inset to panels (j-I): Typical hexagonal lattice electron diffraction patterns of the corresponding 2D sheets. Scale bars: $5 \mathrm{~nm}^{-1}$.

annealing, the Raman spectrum of graphene showed a rather small $I_{D} / I_{G}$ ratio averaging at around 0.065 (Fig. 3a and Supplementary Fig. 26) suggesting low defect density. Further analysis of the $D$ and $D^{\prime}$ peak intensity and $I_{D} / I_{G}$ ratio (see inset of Fig. 3a) revealed that the detected defects could be primarily attributed to the edges of nanosheets ${ }^{29}$. Therefore, the basal plane of graphene remains perfectly crystalline, which is consistent with the non-covalent nature of pyrene functionalisation ${ }^{25}$. The Raman spectrum of the annealed hBN showed the typical $G$ band at around $1364.6 \mathrm{~cm}^{-1}$, corresponding to the $E_{2 g}$ vibration mode (Fig. $3 \mathrm{~b}$ and Supplementary Figs 17 and 18). For quantitative comparison, we also characterised tape-exfoliated hBN flakes from the same bulk precursor. The $G$ peak position was almost unshifted while the full peak width at half maximum (FWHM) increased marginally from the average value of $9.2 \mathrm{~cm}^{-1}$ for the tape-exfoliated samples to $10.3 \mathrm{~cm}^{-1}$ for the flakes derived using our method (Fig. 3c). As in the case of graphene, these values suggest negligible damage introduced during production ${ }^{30}$. Similarly, the tested $\operatorname{MoS}_{2} 2 \mathrm{D}$ sheets showed a Raman spectrum identical to that of a tape-exfoliated reference, confirming no significant damage or phase transformation caused by the processing (Fig. 3d). Moreover, no signs of additional oxidation were detected, as the relative intensity of the Raman band at $820 \mathrm{~cm}^{-1}$ associated with the molybdenum oxide ${ }^{31}$ remained the same (inset of Fig. $3 \mathrm{~d}$ ).

Independently, the electrophoretic mobility ${ }^{32}$ of the 2D sheets in a pH-controlled aqueous suspension was measured. As expected, the non-functionalised reference samples of graphene and $\mathrm{hBN}$ produced by the LPE method (open symbols in Fig. $3 \mathrm{~g}$ ) showed the isoelectric pH factors at around 2 and 4 correspondingly ${ }^{33,34}$. However, the same measurements performed on the 2D sheets exfoliated in our method showed a negative value of the mobility in the entire range of the $\mathrm{pH}$ (solid symbols in Fig. $3 \mathrm{~g}$ ). These results suggest the presence of a negative surface charge introduced by the absorbed pyrene tetra sulfonate. Analysis of the electrophoretic mobility within the framework of the Poisson-Boltzmann theory ${ }^{32}$ gave an estimated surface charge density of $13 \mathrm{mC} \mathrm{m}^{-2}$. This value corresponds to no more than 

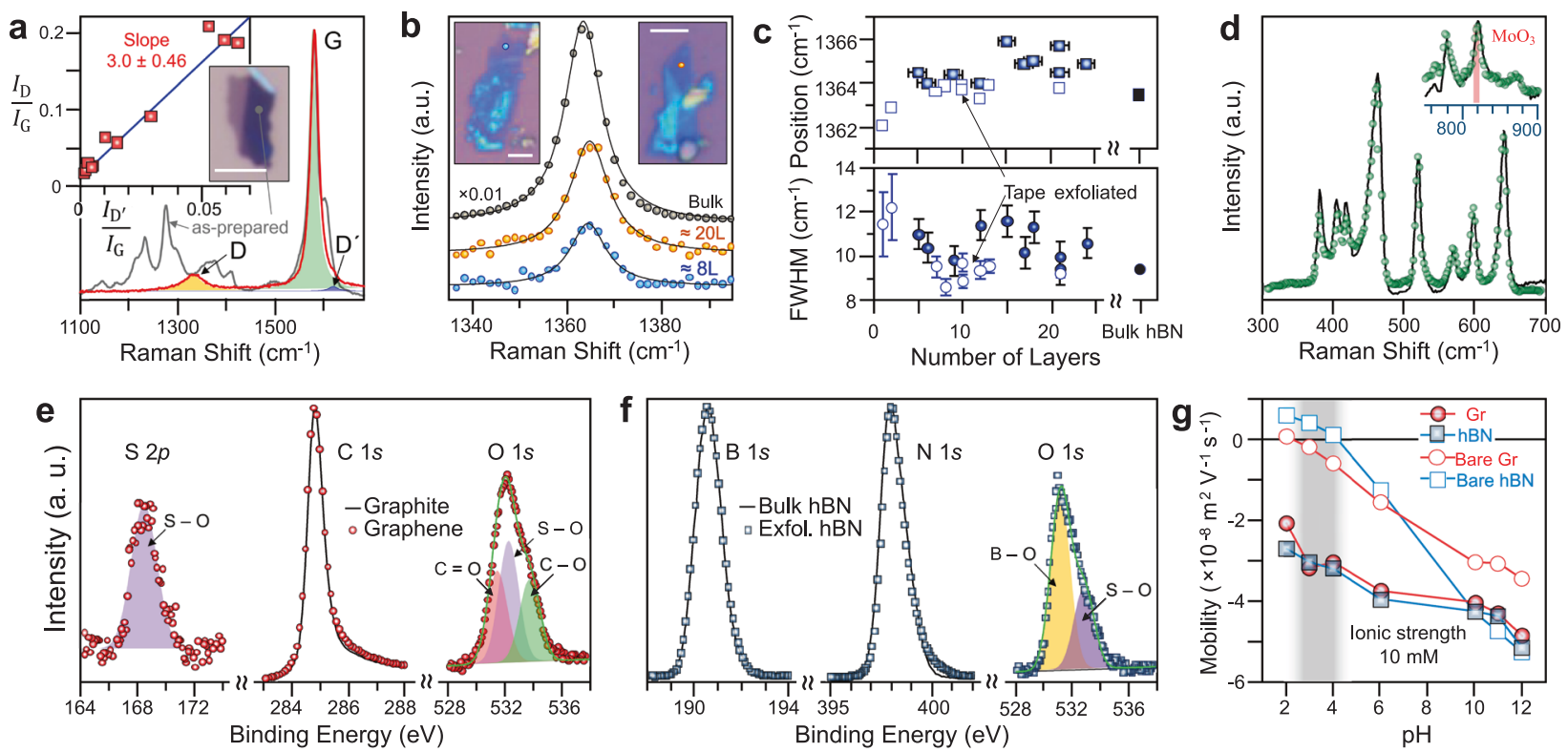

Fig. 3 Crystal quality and non-covalent functionalisation of the 2D sheets. a Raman spectrum of the as-prepared (grey trace) and annealed at $1100{ }^{\circ} \mathrm{C}$ (red trace) graphene $2 \mathrm{D}$ sheets. Inset: Intensity ratio $I_{\mathrm{D}} / I_{\mathrm{G}}$ versus $I_{\mathrm{D} /} / I_{\mathrm{G}}$ measured for the annealed graphene obtained from fitting three Lorentzian functions to the spectrum. The data are approximated by a linear dependence with a slope close to three ${ }^{29}$. $\mathbf{b}$ Raman spectra of bulk hBN and annealed hBN 2D sheets (circles) and the Lorentzian approximation used to find the position and width of the $\mathrm{E}_{2 \mathrm{~g}}$ mode ${ }^{30}$. The micrographs in the insets to $\mathbf{a}, \mathbf{b}$ show the position of the laser spot. Scale bars: $3 \mu \mathrm{m}$. c The comparison of the peak position (squares) and FWHM (circles) as a function of the number of layers for the tape exfoliated (open symbols) and acid-assisted exfoliated 2D sheets (light blue symbols) vs. bulk particles (solid symbols). Error bars represent one standard deviation. d Raman spectra of the tape exfoliated (black trace) and acid-assisted exfoliated 2D sheets (green circles) of $\mathrm{MoS}_{2}$. Inset: The spectrum at around $820 \mathrm{~cm}^{-1}$ attributed to the molybdenum oxide $\mathrm{e}^{31}$. e The XPS spectrum of graphene (red circles) and graphite (black trace) highlighting the sulfur $2 p$, carbon $1 s$, and oxygen $1 s$ levels. $\mathbf{f}$ The XPS spectrum of the exfoliated hBN (black squares) and bulk hBN (black curves) highlighting boron $1 \mathrm{~s}$, nitrogen $1 \mathrm{~s}$, and oxygen $1 \mathrm{~s}$ levels. The oxygen $1 s$ peaks in e, $\mathbf{f}$ were deconvoluted to extract the relative contribution from sulfur bound oxygen. $\mathbf{g}$ Electrophoretic mobility of LPE (open symbols) and acid-assisted exfoliated (solid symbols) for graphene (circles) and exfoliated hBN (squares) in the water at different pH. The vertical grey band highlights the isoelectric region for LPE 2D sheets.

$1.5 \mathrm{wt} \%$ of pyrene sulfonate molecules being bound to the $2 \mathrm{D}$ sheets, which is much smaller than is usually reported for a $2 \mathrm{D}$ material surfactant ${ }^{25}$.

Finally, the non-oxidative nature of the entire exfoliation process was confirmed by X-ray photoelectron spectroscopy (XPS). We compared the carbon, boron, and nitrogen $1 \mathrm{~s}$ core level spectra of the bulk graphite and $\mathrm{hBN}$ with the corresponding exfoliated 2D sheets. These XPS spectra were almost identical signalling no major oxidization (Fig. $3 e, f$ and Supplementary Fig. 27). We also observed the oxygen $1 \mathrm{~s}$ core level, where part of the oxygen content is sulfur-bound and believed to originate from the sulfonic groups introduced by the functionalisation. This was confirmed by detailed deconvolution of the oxygen peak at around $532 \mathrm{eV}$, carried out for graphene and hBN (Fig. 3e, f). Without sulfonic groups, the oxygen content was estimated to be around 2-3 at.\% in both graphene and hBN 2D sheets.

\section{Proposed exfoliation mechanism}

The success of the LPE process relies on the relatively weak out-ofplane van der Waals interlayer bonding ${ }^{35}$. Nevertheless, the stiffness of the layered material has also been found to play a key role and ultimately define the lateral size and thickness ${ }^{18,36}$. For our method, the average aspect ratio appears to be 30 times larger than for the conventional LPE (Fig. $2 g-i)$, which implies a different exfoliation mechanism less dependent on the intrinsic mechanical properties. Along with the larger aspect ratio, the exfoliated 2D sheets tend to form straight edges at angles which are multiples of $30^{\circ}$ (Fig. 4c). This behaviour is more typical for micromechanically cleaved flakes and associated with in-plane fracturing along the main crystallographic directions $10 \overline{1} 0,11 \overline{2} 0$ for graphene or hBN, and $11 \overline{2} 0$ for $\mathrm{MoS}_{2}{ }^{37}$. This process assumes relative insignificance of the out-of-plane bonding and can be explained by the reduced strength of the interlayer interaction caused by the foreign molecular species trapped between the atomic layers.

Indeed, our AFM measurements performed on isolated flakes overestimate the thickness deduced from the optical contrast, and can only match the expected Fresnel-law curves $^{38}$ if a thicker monolayer is assumed (Fig. 4d). The AFM thickness versus the number of layers (Fig. 4e) appears to deviate from the reference tape-exfoliated sample by a factor of 1.7 (5.8 ̊ per monolayer) for graphene and 2.9 (10 ̊ per monolayer) for hBN.

Also, the X-ray diffraction (XRD) data (Fig. 4f, g), points to the presence of a significant fraction of $2 \mathrm{D}$ sheets with larger $d$-spacing than expected for pristine bulk material. That is most obvious for exfoliated hBN, where a series of additional (00/) peaks were observed at smaller diffraction angles. These additional reflections can only be explained in terms of the different stages of molecular intercalation ${ }^{39}$. For the case of hBN, it corresponded to Stage I intercalation, while graphene was found to be Stage II intercalated with the same intercalant size of around 5.7 and $4 \AA$. The two distinct sizes are close to the estimated van der Waals thickness of sulfonated (5.5 $\AA$ ) and bare $\left(3.4 \AA\right.$ ) pyrene molecule, respectively ${ }^{40}$. It is also in a good agreement with the apparent optical contrast data (Fig. $2 \mathrm{~g}$ ) with the thickness being the effective $d$-spacing $\left(d_{\mathrm{hBN}}=c_{0}+d_{\mathrm{i}} \approx\right.$ $9.05 \AA$ and $d_{\text {graphene }}=c_{0}+d_{\mathrm{i}} / 2 \approx 6.2 \AA$, where $c_{0}=3.35 \AA$ and $d_{\mathrm{i}}=5.7 \AA$ ).

In our interpretation, the bulk material undergoes simultaneous intercalation and non-covalent functionalisation while exposed to the mixture of CSA and pyrene. The acid intercalation increases the $d$-spacing sufficiently for the sulfonated pyrene to slide between the layers and form the $\pi-\pi$ bonding. After dilution of the acid, the intercalation is reversed, but the sulfonated pyrene 

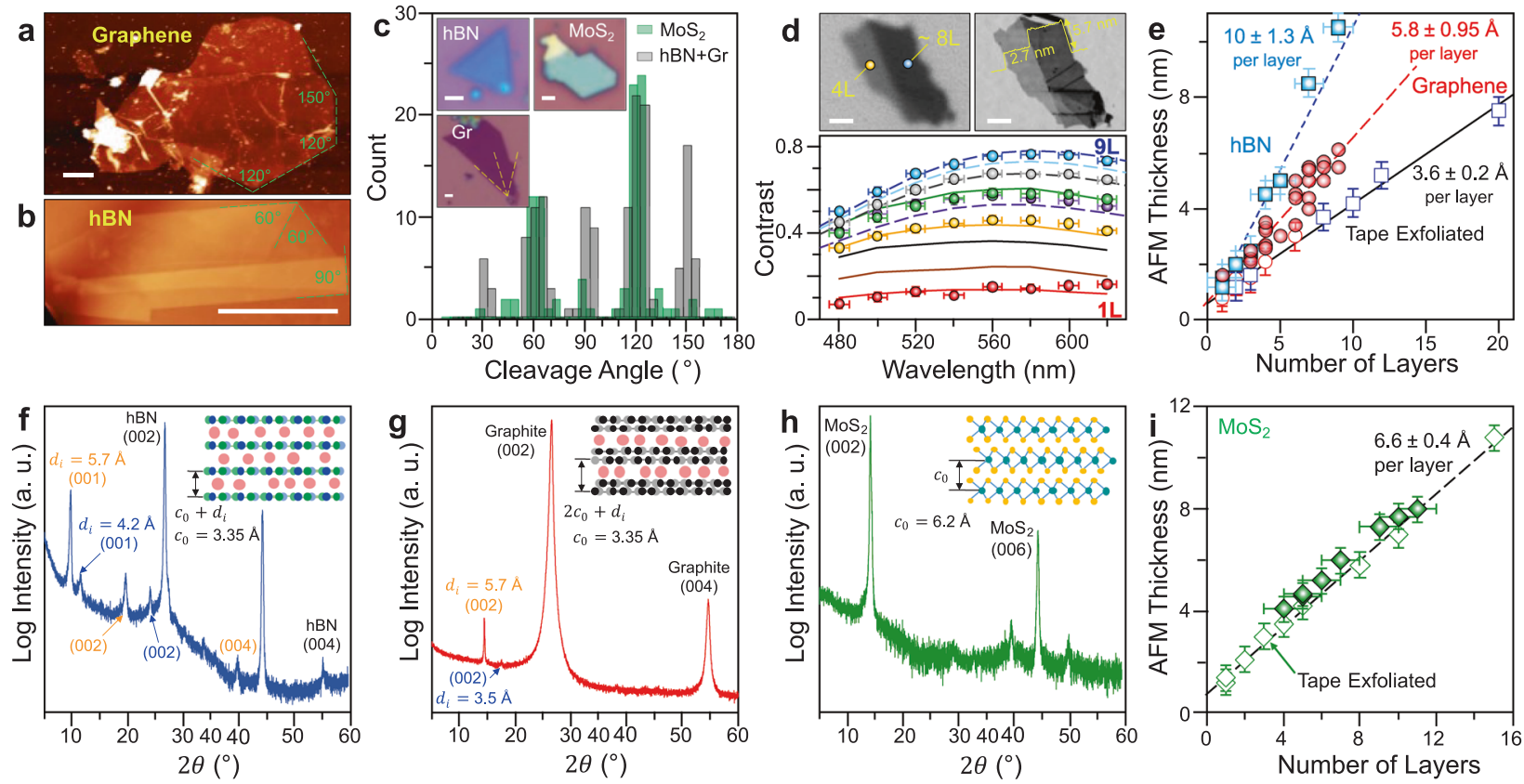

Fig. 4 Signs of the acid intercalation of the 2D sheets. An AFM image of exfoliated graphene (a) and hBN (b) revealing cleaving edges (marked with green dashed lines). c The histogram of the cleaved edge angles for the exfoliated graphene, hBN (grey), and MoS ${ }_{2}$ (green). Insets: Corresponding examples of the exfoliated 2D sheets. $\mathbf{d}$ The wavelength dependence of the optical contrast for tape (solid curves) and acid-assisted (circles) exfoliated graphene 2D sheets with a different number of layers. Error bars represent one standard deviation. Dashed curves represent the prediction of the Fresnel law. Inset: Optical (left) and AFM (right) images of the graphene sheet. Scale bars in a-c: $1 \mu$ m. e AFM thickness versus the number of layers determined from the optical contrast of the tape exfoliated graphene and hBN (open symbols) compared to the acid-exfoliated graphene (solid circles) and hBN (solid squares). The linear regressions determine the effective monolayer thickness. XRD pattern of exfoliated hBN (f) and graphene $(\mathbf{g})$ exhibiting the reflections expected for the bulk crystals (black font) along with the series reflections correspondent to the Stage I in hBN and Stage II in graphene intercalated nanosheets with the effective $d$-spacing, $d=n c_{0}+d_{i}$, where $c_{0}=3.35 \AA, n$ is the stage number and $d_{i}$ is the intercalant size. The reflection at around $44^{\circ}$ originates from the metallic sample holder. $\mathbf{h}$ XRD data for the exfoliated $\mathrm{MoS}_{2}$. i AFM thickness for tape (open symbols) and acid-assisted (solid symbols) exfoliated MoS 2 vs. the number of layers determined from the contrast measurements and corresponding linear regression. Error bars in $\mathbf{e}$ and $\mathbf{i}$ are one standard deviation approximated from AFM height profiles (Supplementary Fig. 10) and Fresnel-law model analysis (Supplementary Figs 11 and 12).

remains attached to the basal planes, and serves as a molecular spacer giving rise to the observed "thickening" of the individual flakes. It is likely that the intercalation is incomplete and does not lead to spontaneous delamination. Instead, it loosens the interlayer binding making the mechanical exfoliation more efficient. Meanwhile, the already present non-covalent functionalisation prevents restacking and ensures the colloidal stability of the nanosheet suspension.

Previous work has shown that the aspect ratio after delamination is proportional to the inverse interlayer binding energy ${ }^{18}$. The van der Waals binding energy, $E_{\mathrm{vdW}}$, scales with the interlayer distance, $d$, as $E_{\mathrm{vdW}} \propto d^{-n}$, where $2 \leq n \leq 4^{32}$. Assuming $n=2$ and the initial van der Waals cut-off distance is $1.65 \AA$, an increase of the $d$-spacing by $5.7 \AA$ reduces $E_{\mathrm{vdW}}$ by more than 20 times, thus increasing the expected aspect ratio by the same amount. This order-of-magnitude estimate is in a reasonable agreement with the observed increase of the aspect ratio shown in Fig. $2 \mathrm{~g}-\mathrm{i}$.

However, our findings for $\mathrm{MoS}_{2}$ seem to contradict this explanation. Both the XRD pattern (Fig. 4g) and the effective monolayer thickness found from the contrast measurements (Fig. 4i) showed no signs of intercalation. It has been reported that acid intercalation in $\mathrm{MoS}_{2}$ and transitional metal dichalcogenides is generally suppressed ${ }^{10}$. Nevertheless, intercalation and subsequent exfoliation may still occur through various in-plane defects, such as rotational misalignment or restacking ${ }^{41}$. This can also explain why the average thickness of $\mathrm{MoS}_{2} 2 \mathrm{D}$ sheets is more than twice that of graphene or $\mathrm{hBN}$. The intercalation takes place only through the in-plane defects, so the thickness of the 2D sheets is defined by the average period of these defects.

\section{Fabrication and electrical characterization of the laminates and coatings}

As an illustration of the quality and processability of these materials, three different applications are demonstrated based on solution-processing of the $2 \mathrm{D}$ sheet suspension or slurry.

Microwave shielding and thermal management applications require flexible, electrically and thermally conductive graphene laminates ${ }^{4}$. Using our graphene $2 \mathrm{D}$ sheets along with vacuum filtration, we fabricated these laminates with a thickness between 10 and $20 \mu \mathrm{m}$, apparent density of $1.9-2.0 \mathrm{~g} \mathrm{~cm}^{-3}$ and a highly aligned in-plane oriented structure (Fig. 5a). The electrical conductivity of the as-prepared laminates was found to be $3100 \pm 250 \mathrm{~S} \mathrm{~cm}^{-1}$. This value is much higher than for the similar graphene-derived films produced by other methods (typically around $100 \mathrm{~S} \mathrm{~cm}^{-1}$ ) and compares well to graphitised $\mathrm{rGO}^{42}$ (Fig. 5b).

The second tested application was the conformal conductive coatings for wearable electronics 5 . To synthesise these, we prepared a high-viscosity binder-free graphene/NMP slurry with a concentration of $50 \mathrm{gl}^{-1}$, suitable for screen printing or blade coating (Fig. 5c). Using this slurry, a polyethylene terephthalate film or regular paper were coated with a $1.4 \mathrm{mg} \mathrm{cm}^{-2}$ graphene layer (Fig. $5 \mathrm{~d}$, e). The dried coating demonstrated excellent surface adhesion and sheet resistance of around $1.38 \mathrm{Ohm} \mathrm{sq}{ }^{-1}$, which sustained $2 \times 10^{4}$ bending cycles with $<1 \%$ change in the resistance (Fig. $5 f$ ).

Additionally, we fabricated heterostructure laminates with both conductive and dielectric layers, such as the two-layer hBN-graphene laminate produced through sequential vacuum filtration (Fig. $5 \mathrm{~g}$ ). The as-prepared dielectric hBN layer exhibited a resistivity of the order of $10^{14} \mathrm{Ohm} \mathrm{cm}$ measured at electric fields up to $2 \mathrm{MV} \mathrm{\textrm {m } ^ { - 1 }}$ 

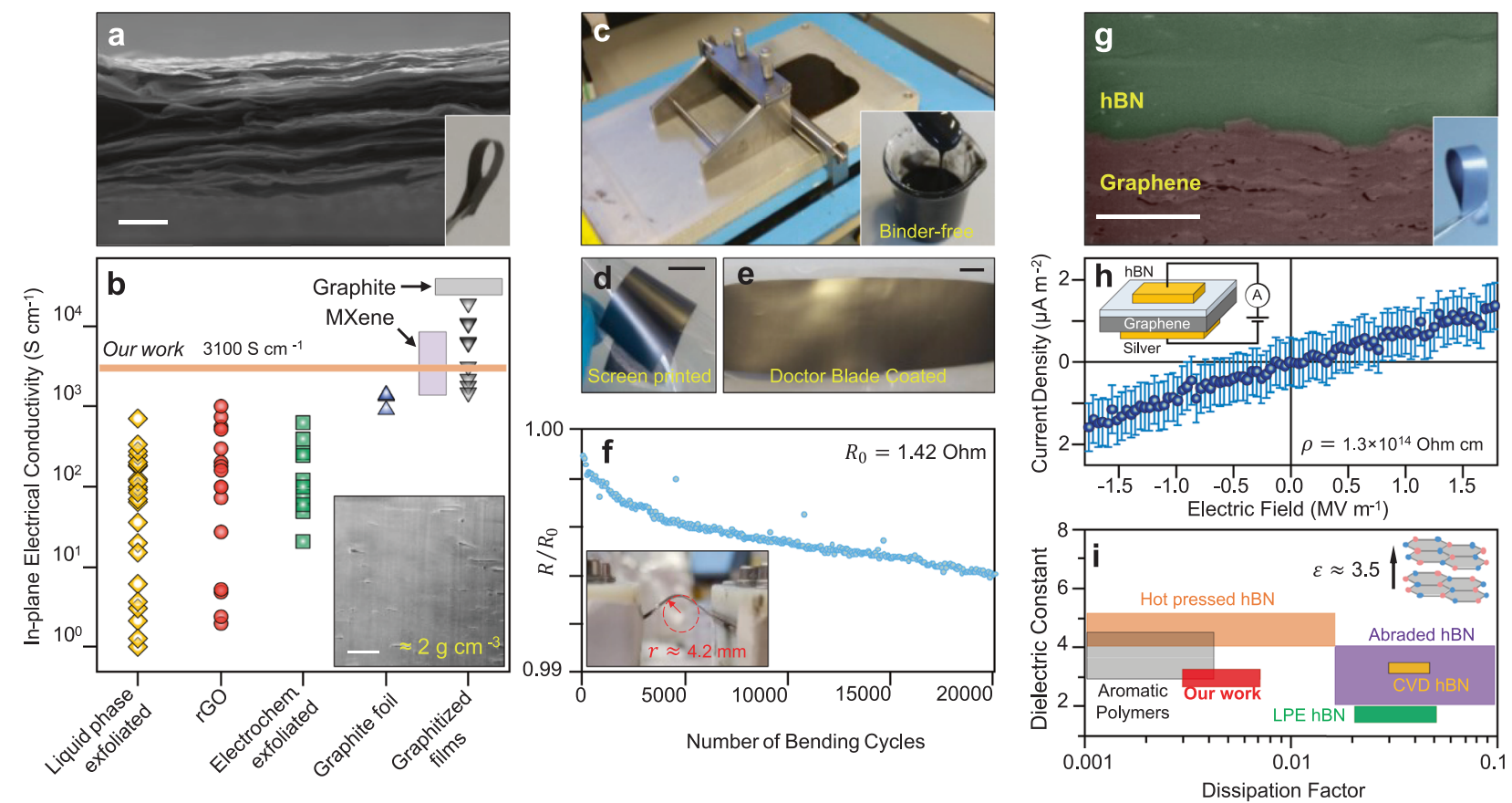

Fig. 5 Example applications for the exfoliated graphene and hBN 2D sheets. a Cross-sectional SEM image of the free-standing graphene laminate. Scale bar: $5 \mu \mathrm{m}$. Inset: Photograph of a bent laminate strip. b Comparison of the in-plane electrical conductivity of our graphene laminate (horizontal orange band marking $3100 \pm 250 \mathrm{~S} \mathrm{~cm}^{-1}$ ) with graphene and MXene laminates ${ }^{51}$, graphitic films ${ }^{42}$, and pyrolytic graphite $^{52}$ (see Supplementary Table 2). Inset: Cross-sectional SEM image of the same laminate prepared using focused ion beam (FIB) milling to resolve the compactness of the sample (density $\approx 2 \mathrm{~g} \mathrm{~cm}^{-3}$ ). Scale bar: $500 \mathrm{~nm}$. c A demonstration of the coating process of a PET film with a binder-free graphene/NMP slurry using conventional doctor blade coater. Inset: Photograph of the graphene/NMP slurry. This slurry can either be screen printed (d) or blade coated (e) on a polymer substrate. Scale bars in d, e: $2 \mathrm{~cm}$. $\mathbf{f}$ Changes in surface resistivity versus the number of bending cycles for a graphene-coated paper (density $1.4 \mathrm{mg} \mathrm{cm}^{-2}$ ). Inset: The graphene-coated paper in the test jig bent to the minimum radius of $4.2 \mathrm{~mm}$. g Colourised cross-sectional SEM image of the hBN/graphene hybrid laminate. The film was cross-sectioned using FIB milling. Scale bar: $5 \mu \mathrm{m}$. Inset: Photograph of a bent $\mathrm{hBN} / \mathrm{graphene}$ laminate strip. $\mathbf{h}$ The current-voltage characteristics of the hBN laminate across its thickness. The electrodes are formed by the bottom graphene laminate on one side, and by a small droplet of a silver adhesive on the other. Error bars are one standard deviation. i Comparison chart of the dielectric constant and dissipation factor of the hBN laminate described in the main text with dielectric properties of different types of dielectrics (see Supplementary Table 3).

(Fig. 5h), which is comparable with the resistivity of hot-pressed hBN ceramics $^{43}$. The low-frequency $(10 \mathrm{kHz})$ dielectric constant of the hBN laminate determined in capacitance measurements was determined to be $\varepsilon=3.3 \pm 0.3$, close to the single-crystal value ${ }^{44}$. The dielectric was also characterised by a surprisingly low value of the loss tangent $\tan \delta \approx 0.003^{45}$ (Supplementary Fig. 30).

In conclusion, we have developed a liquid-phase pathway to effectively exfoliate graphite, $\mathrm{hBN}$ and $\mathrm{MoS}_{2}$ into large few-layered 2D sheets accessing a previously forbidden range of aspect ratios. This was achieved by gentle ultrasound exfoliation assisted by molecular spacers introduced during the chlorosulfuric acid intercalation and in-situ functionalisation. Due to the low defect density and non-oxidative nature of our method, the exfoliated 2D materials demonstrated promising electrical properties. The produced solution-processed graphene laminates exhibited electrical conductivity approaching that of graphitised GO and aromatic polymers, while our dielectric hBN laminates outperform their LPE counterparts and polar polymers. The printed graphene coatings rival the best results reported to date, which makes them especially interesting for the low-cost RFID applications. We believe that other layered materials, which are resistant to acids will be able to be exfoliated and solution-processed in a similar manner.

\section{METHODS}

\section{Intercalation}

In a typical reaction, $1 \mathrm{~g}$ of the layered material (graphite, $\mathrm{hBN}$, or $\mathrm{MoS}_{2}$ ) and $0.1 \mathrm{~g}$ of pyrene (Sigma-Aldrich, $98 \%$ ) were added to a glass flask which has been dehydrated at $70^{\circ} \mathrm{C}$. Then, $20 \mathrm{ml}(50 \mathrm{ml}$ in case of the graphite) of chlorosulfuric acid (Sigma-Aldrich, 99\%) was carefully added to the flask. The mixture was continuously stirred for $48 \mathrm{~h}$ at room temperature. Afterwards, the reaction mixture was diluted by adding $100 \mathrm{ml}$ of deionised water to the acid mixture (Caution: CSA reacts violently with water and releases a copious amount of gas). The resulting mixture was phase-separated in the centrifuge at $10,000 \mathrm{rpm}$ for $10 \mathrm{~min}$. The centrifugation precipitate was rinsed several times with water, $1 \mathrm{M}$ of $\mathrm{KOH}$ (aq.) solution, and then water again. In order to remove the excess sulfonated pyrene, the precipitate was rinsed several times with N-Methyl2-pyrrolidone (NMP) (Sigma-Aldrich) till the separated supernatant becomes clear. In order to improve the purity of the final exfoliated material, NMP can be replaced by dimethyl sulfoxide. The colour of the intercalated hBN powder appears to have a noticeably pink shade probably, due to the optical absorption of sulfonated pyrene molecules absorbed on the surface. Graphene and $\mathrm{MoS}_{2}$ solutions were both dark.

\section{Exfoliation}

Then the pre-intercalated material was dispersed in NMP at a typical concentration of $5 \mathrm{~g} \mathrm{I}^{-1}$. The resulting suspension was sonicated in an ultrasonic bath (Fisherbrand, FB15048) for $10 \mathrm{~min}$ followed by $15 \mathrm{~min}$ homogenising mixing using a high-shear mixer (L5M-A, Silverson Machines Ltd.) at $10,000 \mathrm{rpm}$. The dispersions were left to settle for $48 \mathrm{~h}$ then centrifuged at $1000 \mathrm{rpm}$ for $15 \mathrm{~min}$. The resultant supernatant is the desired suspension of the exfoliated materials.

In order to calculate the exfoliation yield, a known amount of dispersion was filtered through a nylon membrane (pore size of $200 \mathrm{~nm}$ ). The membrane was then soaked in and washed with hot water to remove NMP residue and then dried on a hot plate at $150^{\circ} \mathrm{C}$ for an hour. The mass of flakes was estimated by measuring the membrane weight before and after filtration, which gave the concentration of nanosheets in final dispersion, 
$C_{1}$. Given that the initial concentration, $C_{i}$ of the non-exfoliated material in the dispersion is a known value, the resulting yield is $\frac{C_{1}}{C_{i}}$.

\section{Laminate fabrication}

The free-standing laminates were produced by vacuum filtration through a fine-pore nylon membrane (Whatman, pore size: $0.2 \mu \mathrm{m}$ ). After filtration, the membrane was rinsed in hot water for $30 \mathrm{~min}$ and dried on a hot plate for $1 \mathrm{~h}$ at $150^{\circ} \mathrm{C}$. In order to improve mechanical cohesion, laminates were compressed using a hydraulic press machine at 350 bar. The membrane was dissolved in formic acid, and the resulting free-standing films were washed with water and dried in an oven $\left(70^{\circ} \mathrm{C}\right)$ overnight. Binder-free graphene ink was prepared by successive centrifugation of the initial dispersion to increase the concentration to about $50 \mathrm{gl}^{-1}$.

\section{Characterization}

Scanning electron microscopy (SEM) measurements of cross-sections were performed using a Tescan Mira 3 FEG-SEM. The operating voltage and working distance were $15 \mathrm{kV}$ and $10 \mathrm{~mm}$, respectively. Some of graphene and graphene/hBN hybrid films were cut and imaged by focused ion beam (FIB) milling using FEI HELIOS 660i Nanolab FIB. X-ray diffraction data were collected on a Rigaku SmartLab diffractometer using $\mathrm{Cu}$ Ka radiation and parallel beam optics over the range of $5^{\circ} \leq 2 \theta \leq 90^{\circ}$ for $1 \mathrm{~h}$. UV-Vis spectroscopy (Agilent, Cary 5000) was used to measure absorbance spectra of nanosheets dispersions in NMP or water. X-ray photoelectron spectroscopy (XPS) was conducted using a Thermo Scientific ESCALab 250Xi Spectrometer. Transmission electron microscopy (TEM) was performed using TALOS F200X TEM. To produce suspended samples for TEM a droplet of the dispersions containing 2D sheets $\left(\approx 50 \mathrm{mg} \mathrm{I}^{-1}\right)$ was placed on a copper TEM grid with a lacey carbon support film (Agar Scientific) and left to dry at $70^{\circ} \mathrm{C}$ overnight. Raman spectra were captured using a Renishaw inVia with laser excitation energy of $1.96 \mathrm{eV}(633 \mathrm{~nm})$. The measurements were recorded with a $100 \times$ lens and a 1200 -groove $\mathrm{mm}^{-1}$ grating.

The dielectric properties of hBN films were measured by an LCR meter (HM8118 LCR Bridge/Meter, Hameg Instruments GmbH, Germany) in the air at ambient temperature. Average capacitance within $0.1-10 \mathrm{kHz}$ for at least three different measurements was used to determine the capacitance. The dielectric constant was then calculated based on the thickness measured using SEM.

The surface resistivity of the graphene laminates was measured by the van der Pauw method. The bulk electrical resistivity was given by the product of the slope of the voltage against current plot and the samples' dimensions.

Charging properties of the exfoliated flakes were studied using electrophoresis. All the measurements were performed with a ZetaNano ZS (Malvern Instruments, UK) at room temperature. NMP was replaced with water, and the particle concentration was adjusted to about $20-50 \mathrm{mg} \mathrm{I}^{-1}$. The ionic strength of the dispersions was adjusted to $10 \mathrm{mM}$, and $\mathrm{pH}$ varied between 2-12 by adding an appropriate amount of $\mathrm{NaCl}, \mathrm{NaOH}$, or $\mathrm{HCl}$. In order to compare the charging properties of bare particles, layered materials of the same source were exfoliated in NMP using one-hour bath sonication and left it overnight to separate large pieces. These $2 \mathrm{D}$ sheets were also redispersed in water by solvent exchange.

The lateral size (square root of the area) and the number of layers of the 2D sheets were determined using a combination of AFM imaging (Dimension 3100, Bruker) and optical microscopy (Nikon Eclipse LV100ND). The optical contrast of the $2 \mathrm{D}$ sheets deposited atop of a $\mathrm{Si} / \mathrm{SiO}_{2}$ wafer was quantified using a monochromic camera (Nikon DS-Qi2). Images were acquired under filtered light using a set of narrow-band filters (Thor's lab, FWHM of $10 \pm 2 \mathrm{~nm}$ ). The exact number of layers in each nanosheet was determined using a Fresnel-law-based model ${ }^{38}$. The AFM image of the same $2 \mathrm{D}$ sheets was then captured. As a reference, we measured the optical contrast and AFM thickness of tape-exfoliated 2D sheets deposited onto the same silicon wafers (see Supplementary Figs 5-17).

The data that support the findings of this study are available from the corresponding author upon reasonable request.

Received: 25 October 2020; Accepted: 4 February 2021; Published online: 06 April 2021

\section{REFERENCES}

1. Lin, L., Peng, H. \& Liu, Z. Synthesis challenges for graphene industry. Nat. Mater. 18, 520-524 (2019)
2. Sun, P., Wang, K. \& Zhu, H. Recent developments in graphene-based membranes: structure, mass-transport mechanism and potential applications. Adv. Mater. 28, 2287-2310 (2016).

3. Ries, L. et al. Enhanced sieving from exfoliated $\mathrm{MoS}_{2}$ membranes via covalent functionalization. Nat. Mater. 18, 1112-1117 (2019).

4. Fu, Y. et al. Graphene related materials for thermal management. 2D Mater. 7 , 012001 (2019).

5. Wang, C. et al. Advanced carbon for flexible and wearable electronics. Adv. Mater. 31, 1801072 (2019)

6. Marcano, D. C. et al. Improved synthesis of graphene oxide. ACS Nano 4, 4806-4814 (2010).

7. Dreyer, D. R., Park, S., Bielawski, C. W. \& Ruoff, R. S. The chemistry of graphene oxide. Chem. Soc. Rev. 39, 228-240 (2010).

8. Coleman, J. N. et al. Two-dimensional nanosheets produced by liquid exfoliation of layered materials. Science 331, 568-571 (2011).

9. Paton, K. R. et al. Scalable production of large quantities of defect-free few-layer graphene by shear exfoliation in liquids. Nat. Mater. 13, 624-630 (2014).

10. McKelvy, M. J. \& Glaunsinger, W. S. Molecular intercalation reactions in lamellar compounds. Annu. Rev. Phys. Chem. 41, 497-523 (1990).

11. Shih, C. J. et al. Bi- and trilayer graphene solutions. Nat. Nanotechnol. 6, 439-445 (2011).

12. Fan, X. et al. Controlled exfoliation of $\mathrm{MoS}_{2}$ crystals into trilayer nanosheets. J. Am. Chem. Soc. 138, 5143-5149 (2016).

13. Lin, Z. et al. Solution-processable 2D semiconductors for high-performance largearea electronics. Nature 562, 254-258 (2018).

14. Peng, J. et al. Very large-sized transition metal dichalcogenides monolayers from fast exfoliation by manual shaking. J. Am. Chem. Soc. 139, 9019-9025 (2017).

15. Kim, J. et al. Extremely large, non-oxidized graphene flakes based on spontaneous solvent insertion into graphite intercalation compounds. Carbon 139, 309-316 (2018).

16. Abdelkader, A. M., Cooper, A. J., Dryfe, R. A. W. \& Kinloch, I. A. How to get between the sheets: a review of recent works on the electrochemical exfoliation of graphene materials from bulk graphite. Nanoscale 7, 6944-6956 (2015).

17. Parvez, K., Yang, S., Feng, X. \& Müllen, K. Exfoliation of graphene via wet chemical routes. Synth. Met. 210, 123-132 (2015).

18. Backes, C. et al. Equipartition of energy defines the size-thickness relationship in liquid-exfoliated nanosheets. ACS Nano 13, 7050-7061 (2019).

19. Davis, V. A. et al. True solutions of single-walled carbon nanotubes for assembly into macroscopic materials. Nat. Nanotechnol. 4, 830-834 (2009).

20. Cremlyn, R. J. W. Chlorosulfonic Acid: A Versatile Reagent. (Royal Society of Chemistry, 2002).

21. Behabtu, N. et al. Spontaneous high-concentration dispersions and liquid crystals of graphene. Nat. Nanotechnol. 5, 406-411 (2010).

22. Jasuja, K. et al. Introduction of protonated sites on exfoliated, large-area sheets of hexagonal boron nitride. ACS Nano 12, 9931-9939 (2018).

23. David, L., Bhandavat, R. \& Singh, G. $\mathrm{MoS}_{2}$ /graphene composite paper for sodiumion battery electrodes. ACS Nano 8, 1759-1770 (2014).

24. Pagona, G., Bittencourt, C., Arenal, R. \& Tagmatarchis, N. Exfoliated semiconducting pure $2 \mathrm{H}-\mathrm{MoS}_{2}$ and $2 \mathrm{H}-\mathrm{WS}_{2}$ assisted by chlorosulfonic acid. Chem. Commun. 51, 12950-12953 (2015).

25. Parviz, D. et al. Dispersions of non-covalently functionalized graphene with minimal stabilizer. ACS Nano 6, 8857-8867 (2012).

26. Harvey, A. et al. Non-resonant light scattering in dispersions of $2 \mathrm{D}$ nanosheets. Nat. Commun. 9, 4553 (2018).

27. Kouroupis-Agalou, K. et al. Fragmentation and exfoliation of 2-dimensional materials: a statistical approach. Nanoscale 6, 5926-5933 (2014).

28. Schlierf, A. et al. Nanoscale insight into the exfoliation mechanism of graphene with organic dyes: effect of charge, dipole and molecular structure. Nanoscale $\mathbf{5}$, 4205-4216 (2013).

29. Eckmann, A. et al. Probing the nature of defects in graphene by Raman spectroscopy. Nano Lett. 12, 3925-3930 (2012).

30. Nemanich, R. J., Solin, S. A. \& Martin, R. M. Light scattering study of boron nitride microcrystals. Phys. Rev. B 23, 6348-6356 (1981).

31. Windom, B. C., Sawyer, W. G. \& Hahn, D. W. A Raman spectroscopic study of MoS2 and MoO3: applications to tribological systems. Tribol. Lett. 42, 301-310 (2011).

32. Israelachvili, J. N. Intermolecular and Surface Forces. (Academic Press, 2011).

33. Koestner, R., Roiter, Y., Kozhinova, I. \& Minko, S. Effect of local charge distribution on graphite surface on Nafion polymer adsorption as visualized at the molecular level. J. Phys. Chem. C 115, 16019-16026 (2011).

34. Xiong, C. \& Tu, W. Synthesis of water-dispersible boron nitride nanoparticles. Eur. J. Inorg. Chem. 2014, 3010-3015 (2014).

35. Mounet, N. et al. Two-dimensional materials from high-throughput computational exfoliation of experimentally known compounds. Nat. Nanotechnol. 13, 246-252 (2018). 
36. Li, Z. et al. Mechanisms of liquid-phase exfoliation for the production of graphene. ACS Nano 4, 10976-10985 (2020).

37. Guo, Y. et al. Distinctive in-plane cleavage behaviors of two-dimensional layered materials. ACS Nano 10, 8980-8988 (2016).

38. Gorbachev, R. V. et al. Hunting for monolayer boron nitride: optical and Raman signatures. Small 7, 465-468 (2011).

39. Dresselhaus, M. S. \& Dresselhaus, G. Intercalation compounds of graphite. Adv. Phys. 30, 139-326 (1981).

40. Jensen, J. H. \& Kromann, J. C. The molecule calculator: a web application for fast quantum mechanics-based estimation of molecular properties. J. Chem. Educ. 90, 1093-1095 (2013).

41. Shiojiri, M., Isshiki, T., Saijo, H., Yabuuchi, Y. \& Takahashi, N. Cross-sectional observations of layer structures and stacking faults in natural and synthesized molybdenum disulfide crystals by high-resolution transmission electron microscopy. J. Electron Microsc. 42, 72-78 (1993).

42. Chen, X. et al. Graphitization of graphene oxide films under pressure. Carbon 132, 294-303 (2018).

43. Britnell, L. et al. Electron tunneling through ultrathin boron nitride crystalline barriers. Nano Lett. 12, 1707-1710 (2012).

44. Ahmed, F. et al. Dielectric dispersion and high field response of multilayer hexagonal boron nitride. Adv. Funct. Mater. 28, 1804235 (2018).

45. Kelly, A. G., Finn, D., Harvey, A., Hallam, T. \& Coleman, J. N. All-printed capacitors from graphene-BN-graphene nanosheet heterostructures. Appl. Phys. Lett. 109, 023107 (2016).

46. Yang, $H$. et al. A simple method for graphene production based on exfoliation of graphite in water using 1-pyrenesulfonic acid sodium salt. Carbon 53, 357-365 (2013).

47. Chen, S. et al. Simultaneous production and functionalization of boron nitride nanosheets by sugar-assisted mechanochemical exfoliation. Adv. Mater. 31, 1804810 (2019).

48. Lee, D. et al. Scalable exfoliation process for highly soluble boron nitride nanoplatelets by hydroxide-assisted ball milling. Nano Lett. 15, 1238-1244 (2015).

49. Worsley, R. et al. All-2D material Inkjet-printed capacitors: toward fully printed integrated circuits. ACS Nano 13, 54-60 (2019).

50. Kelly, A. G. et al. All-printed thin-film transistors from networks of liquid-exfoliated nanosheets. Science 356, 69-73 (2017).

51. Han, M. et al. Beyond $\mathrm{Ti}_{3} \mathrm{C}_{2} \mathrm{~T}_{\mathrm{x}}: \mathrm{MXenes}$ for electromagnetic interference shielding. ACS Nano 14, 5008-5016 (2020).

52. Song, R. et al. Flexible graphite films with high conductivity for radio-frequency antennas. Carbon 130, 164-169 (2018).

\section{ACKNOWLEDGEMENTS}

M.M.G. acknowledges funding from the Swiss National Science Foundation (Project 174952). S.J.H. acknowledges funding from bp-ICAM, the Engineering and Physical Sciences Research Council (UK) EPSRC (grants EP/M010619/1, EP/S021531/1, EP/ P009050/1) and the European Commission H2020 ERC Starter grant EvoluTEM (715502). This work was supported by the Henry Royce Institute for Advanced Materials, funded through EPSRC grants EP/R00661X/1, EP/S019367/1, EP/P025021/1, EP/P025498/1 and the Centre for Doctoral Training (CDT) Graphene-NOWNANO.
A.V.K. also acknowledges support from EPSRC (grant EP/V036343/1) and from the European Graphene Flagship Project.

\section{AUTHOR CONTRIBUTIONS}

M.M.G., K.S.N., and A.V.K. conceived the study. M.M.G. developed the chemical method, prepared the samples and performed UV-Vis spectroscopy and electrophoresis measurements. M.M.G. and B.M. performed the Raman analysis. M.A. with the help of M.M.G., M.S., X.Z., and S.J.H. performed XRD, SEM, and TEM analysis. X. Z. performed FIB. G.P. performed the electrical characterizations. M.M.G. with the input from R.G. performed AFM and optical microscopy. J.G. performed XPS analysis. T.G. prepared the raw precursor materials. M.M.G. and A.V.K. analysed and interpreted the data and wrote the manuscript with the input from all co-authors.

\section{COMPETING INTERESTS}

The authors declare no competing interests.

\section{ADDITIONAL INFORMATION}

Supplementary information The online version contains supplementary material available at https://doi.org/10.1038/s41699-021-00215-2.

Correspondence and requests for materials should be addressed to A.V.K.

Reprints and permission information is available at http://www.nature.com/ reprints

Publisher's note Springer Nature remains neutral with regard to jurisdictional claims in published maps and institutional affiliations.

\section{(i)}

Open Access This article is licensed under a Creative Commons Attribution 4.0 International License, which permits use, sharing, adaptation, distribution and reproduction in any medium or format, as long as you give appropriate credit to the original author(s) and the source, provide a link to the Creative Commons license, and indicate if changes were made. The images or other third party material in this article are included in the article's Creative Commons license, unless indicated otherwise in a credit line to the material. If material is not included in the article's Creative Commons license and your intended use is not permitted by statutory regulation or exceeds the permitted use, you will need to obtain permission directly from the copyright holder. To view a copy of this license, visit http://creativecommons. org/licenses/by/4.0/.

(c) The Author(s) 2021 